\title{
AN INTEGRATED LOW-COST RADIATION DOSIMETER UTILIZING MICROORGANISM AS RADIATION-SENSITIVE MATERIAL
}

\author{
C.K. Yoon", M. Ochoa, A. Kim, R. Rahimi and B. Ziaie \\ Purdue University, West Lafayette, Indiana, USA
}

\begin{abstract}
We present a simple, low-cost, and robust radiation dosimeter that uses a microorganism (yeast) as the radiation sensing material. The radiation damage to the yeast is used to modulate the impedance of a flexible sensor in which a yeast-glucose film constitutes the dielectric between two metallic films. Adding DI water to the sensor after radiation exposure results in the initiation of the fermentation process, the output of which is monitored by measuring the impedance between the electrodes. Radiation damaged yeast cells result in a reduced $\mathrm{CO}_{2}$ formation, a decreased total amount of carbonic ions in the fermenting solution, and a drop in the electrical conductivity of the medium between the two electrodes. This is the first reported radiation sensor which utilizes microorganism as the radiation-sensitive material, offering a direct biological correlate to the radiation-induced DNA damage. A prototype sensor with dimensions of $18 \times 18 \mathrm{~mm}^{2}$ offers a maximum sensitivity of $0.154 \Omega / \Omega_{0}$ decade-rad and an average resolution of $5 \mathrm{rad}$ during the first $10 \mathrm{rad}$ of exposure.
\end{abstract}

\section{INTRODUCTION}

Ionizing radiations are commonly used/encountered in medical imaging, radiation therapy, nuclear power plants, food sterilization, and scientific research. Although the effects of high dose radiation exposure (Hiroshima, Chernobyl, and Fukushima) is well documented [1], long term exposure to lower levels used in medical imaging and other civilian application can also pose significant health risks [2]. Personnel monitoring dosimeters in various forms and shapes have been utilized to track workers in high-risk sites (e.g., hospitals, laboratories, power plants) [3]. Some of these such as film badges [4] and can be considered as the early versions of the wearable devices. More recently, solid-state diodes and RADFETS have made significant penetration into the personnel monitoring market $[5,6]$. These devices, however, are expensive and many of them have to be shipped to the manufacturer for readout. Moreover, they only measure the number of ions generated via radiation on their (non-biological) sensing material. As such, they are not a direct indication of the biological damage. A more clinically-relevant dosimeter should be able to measure biological changes in response to radiation in order to better quantify the effective radiation dosage to human tissues and organs.

Living matter undergoes physical (sometimes lethal) damage to their DNA when exposed to the ionizing radiation [7]. If such physiochemical alterations to the DNA can be utilized to measure the absorbed dosage, it can provide an invaluable information regarding the biological damage to the organism. One particular organism that can serve such purpose (radiation surrogate) is the yeast, a eukaryotic microorganism with wide and ancient commercial applications in food and beverage industry. Yeast is a well-studied microorganism having homologous genetic sequence to humans $[8,9]$. Moreover, it is a robust material $\left(50{ }^{\circ} \mathrm{C},>250\right.$ $\mathrm{MPa}, 5-8 \%$ humidity) with a long shelf life [10, 11]. Among different yeasts, Saccharomyces cerevisiae yeast has been among the most studied, due to its genomic similarity to mammalian cells. Researchers have also investigated its response to the radiation, according to which, yeast is found to be semi-resistant to ionizing radiation, i.e., not all yeast cells are completely inactivated by a given dose. Rather, radiation exposure to a population of yeast cells impairs their average metabolic activity $[12,13]$.

To facilitate personal radiation exposure measurements which are more indicative of biological damage, we have developed a lowcost, film-type, disposable radiation dosimeter that utilizes yeast as the sensing material. During the exposure, high energy ionizing radiation damages the DNA chain, inducing lethal or sub-lethal damage to the yeast cells, hence, interfering with their metabolic activity. This can be quantified by monitoring the fermentation rate after exposure to the radiation through the measurement of the produced $\mathrm{CO}_{2}$ and formation of carbonic acid byproducts.

\section{DESIGN}

The schematic and operation principle of the dosimeter is illustrated in Figure 1. The simple but robust film type design is based on aluminum film and double-sided tape. Two aluminum pieces are used to create two parallel-plate electrodes sandwiching a bilayer of two tapes, one coated with yeast and the other with glucose. The tape is patterned with holes to reduce the electrical impedance between the two aluminum electrodes. For measurements, the film is activated by the introduction of moisture (DI water) initiating the fermentation process with ethanol and $\mathrm{CO}_{2}$ as the byproducts. The $\mathrm{CO}_{2}$ is then dissolved in the water up to its maximum solubility level (after which bubbles are generated); as it dissolves it creates carbonic acid, which decreases the electrical impedance between the electrodes due to the increase in charge carriers (i.e., $\mathrm{H}^{+}$and $\mathrm{HCO}_{3}{ }^{-}$ions), Figure $1 \mathrm{~b}$. If the film has not been exposed to radiation, then fermentation (and $\mathrm{CO}_{2}$ dissolution) proceeds at its normal rate, with electrical impedance decreasing rapidly over time. In contrast, if the film has been radiated, the fermentation rate is reduced due to radiation-induced damage to yeast cells; as a result, the rate of $\mathrm{CO}_{2}$ dissolution is lower, resulting in a lower rate of change of electrical impedance. Thus, the rate of change of impedance (up to the solubility level of $\mathrm{CO}_{2}$ ) is representative of the radiation dose to which the yeast are exposed.

\section{FABRICATION}

The sensor is fabricated using a rapid prototyping technique and layer-by-layer assembly, Figure 2. First, two metal electrodes are created by laser-machining aluminum tape into " $\mathrm{P}$ " shape (electrode area $=10 \times 10 \mathrm{~mm}^{2}$ ) using a $1.06 \mu \mathrm{m}$-wavelength fiber laser engraving system (PLS6MW, Universal Laser Systems, Inc.), Figure 2a. Next, a layer of double-sided tape $\left(\operatorname{Scotch}^{\mathbb{R}}\right.$ double sided tape, $3 \mathrm{M})$ is laser machined with a $\mathrm{CO}_{2}$ laser $(10.6 \mu \mathrm{m})$ to create a square with a $5 \times 5$ array of millimeter-diameter holes. The holes are one millimeter apart from each other. The holes enable electrical conduits between aluminum electrodes, Figure 2b. Freezer paper is also laser machined into squares of $18 \times 18 \mathrm{~mm}^{2}$ and used to enclose the materials into a film shape by the lamination process (the hydrophobic side of the freezer paper is used for lamination to seal and package the device at the end). To assemble the sensor, each aluminum tape is sandwiched between a layer of freezer paper (hydrophobic side) and the laser-machined tape layer as shown in Figure 2c. The tape layers are then plasma-treated to improve hydrophilicity for enhanced water penetration/spreading on the entire surface area of the double-sided tape, Figure 2d. 
(a) Disassembled device schematic

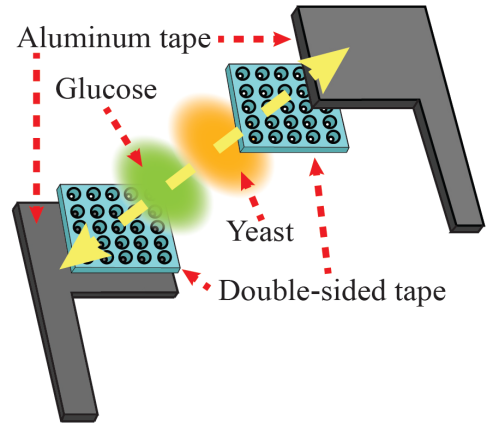

(b) Cross-section of the device

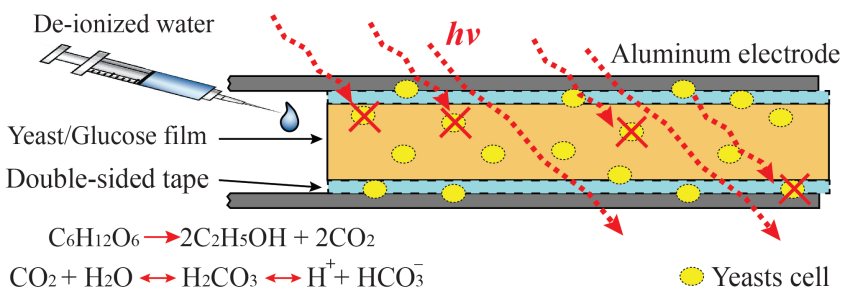

Figure 1: (a) Illustration of the disassembled sensor structure and cross-section of the device. (b) Fraction of viable yeasts (yellow) is reduced after the radiation exposure (crossed out with red X). Carbonic acid produced by fermenting live yeasts contributes to the decreased resistivity between the electrodes.

After plasma treatment, $10 \mathrm{mg}$ of yeast powder (Fleischmann's ${ }^{\mathbb{B}}$ Instant Dry ${ }^{\mathbb{B}}$ ) is screen-printed onto the doublesided tape, Figure 2e. The process is repeated on the other tape using $10 \mathrm{mg}$ glucose particles (D-(+) Glucose $\geq 99.5 \%$, Sigma-Aldrich ${ }^{\circledR}$ Co.). Both yeast and glucose powders are ground to make them fine enough for uniform coating to the double sided tape. The outer boundaries of the symmetric freezer papers are then aligned with each other enclosing the double sided layers with yeast and glucose inside, and the sensor is sealed by thermal lamination as shown in Figure $2 \mathrm{f}$. The lamination temperature was controlled to be $380{ }^{\circ} \mathrm{C}$ and only applied at the edges to avoid damaging yeast since the yeast can burn inside the sensor at such high temperatures. All edges of the freezer paper are laminated except for one side, where aluminum electrodes are wired out for electrical measurement. This opening is also where water is injected to initiate the fermentation. Fabricated samples are kept refrigerated $\left(3^{\circ} \mathrm{C}\right)$ when not in use to prolong their shelf life. Figure 3(a) is an image of an open sensor just prior to final packaging. Figure 3(b), shows a packaged one, ready to use.

\section{EXPERIMENTAL SETUP}

Prior to the radiation exposure, all samples and de-ionized water were kept in refrigerated conditions. To evaluate the effect of radiation on the yeast viability and hence the transducer output, various sensors ( 5 samples in each group) were exposed to different doses of radiation $(0,10,100$ and $1000 \mathrm{rad})$ using a Co-60 $(1.13 \mathrm{MeV})$ source. After exposure, each sensor was injected with $0.1 \mathrm{~mL}$ of de-ionized water using a $30 \mathrm{G}$ hypodermic needle (yeast concentration inside the sensor to $100 \mathrm{~g} / \mathrm{L}$ ) [14]. The sensors were then connected directly to an LCR meter (LCR-821, GW Instek), and the electrical impedance $(\mathrm{Z}=\mathrm{R}+\mathrm{jX})$ was measured over time (30 minutes) at the frequency of $1 \mathrm{kHz}$.

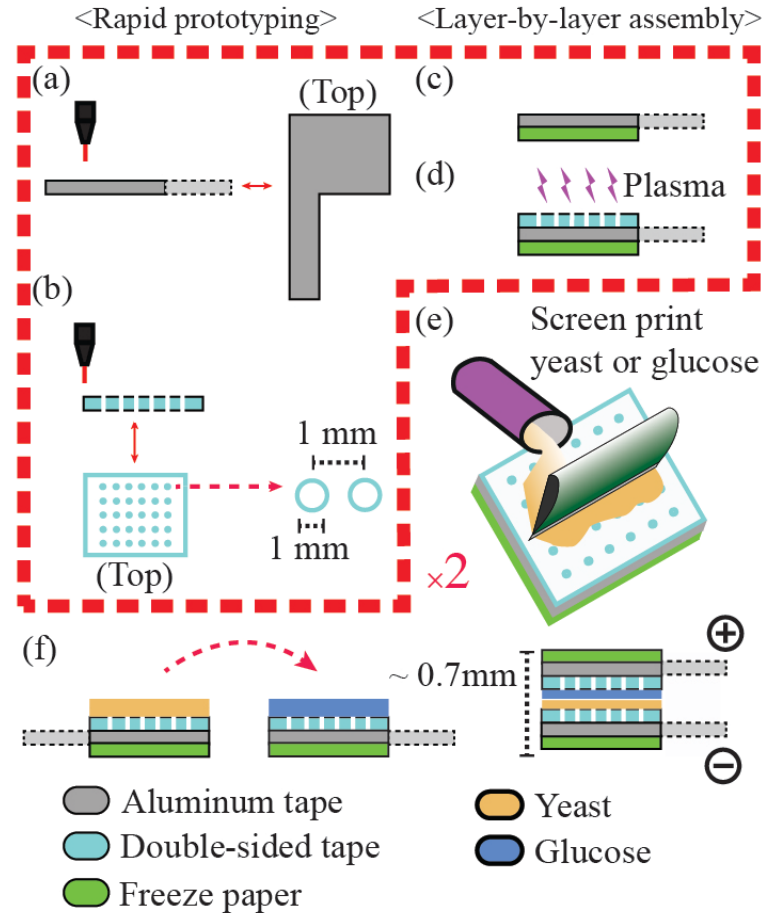

Figure 2. Fabrication process of the film radiation sensor. (a-b) laser machine aluminum $\left(10 \times 10 \mathrm{~mm}^{2}\right)$ and tape $(5 \times 5$ array that are a millimeter apart from each other) using laser engraver; (c) assemble tape and aluminum on hydrophobic side of freezer paper substrate; (d) plasma treat the double sided layer surface to increase the hydrophilicity; (e) deposit yeast powder on top of the double-sided tape side, then repeat the process through $(a-d)$ to make a symmetric glucose layer; (f) sandwich tapes together and seal edges of the freezer paper to package with thermal lamination except the open-end at electrical connection side.

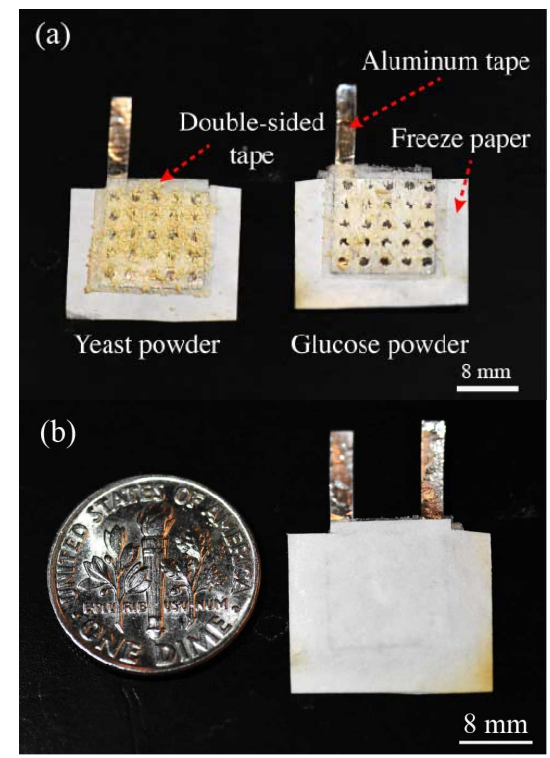

Figure 3. (a) A photograph showing the inside view of the sensor, (b) image of film radiation sensor after lamination. 
Commercial baker's yeast (as used in this experiment) can contain other unknown elements (e.g., remnants from nutrients used during culturing) that could change the electrical response during the experiment. To evaluate the effect of such contaminants, we conducted a control experiment by measuring the impedance over time of a sensor maintained at $3{ }^{\circ} \mathrm{C}$ for at least 10 minutes; at this temperature yeast remained inactive and the change in resistance was only be the result of the dissolved impurities.

\section{RESULTS AND DISCUSSION}

Figure 4 shows the normalized impedance change as a function of time after the activation of the irradiated sensors by adding DI water. The results show two operation regimes. The first one (0-2 minutes) exhibits a drastic drop in impedance within a short time (large negative slope). The second phase, in contrast, shows a more constant impedance over time (zero or slightly negative slope). The two regimes can be attributed to the generation and dissolution of $\mathrm{CO}_{2}$ during fermentation. Immediately after the onset of fermentation, any $\mathrm{CO}_{2}$ that is generated is quickly dissolved in the water, creating carbonic acid (and an increase in charge carriers, $\mathrm{H}^{+}$ and $\mathrm{HCO}_{3}{ }^{-}$ions). This condition remains true until the concentration of $\mathrm{CO}_{2}$ reaches the maximum solubility in water (1.5 $\mathrm{g} \mathrm{CO}_{2} / \mathrm{kg} \mathrm{H}_{2} \mathrm{O}$ ) [15]. The transition time can be predicted based on the generation rate of $\mathrm{CO}_{2}$ as a function of the amount of liquid and yeast in the chamber (assuming glucose is sufficient not be a limiting factor). Previous experiments have revealed a $\mathrm{CO}_{2}$ generation rate of approximately $5 \mathrm{~mL} \mathrm{CO} / \mathrm{min}$ using $3 \mathrm{~g}$ of yeast [14]; when scaled to the amount of yeast in the present device $(0.01 \mathrm{mg})$, the rate becomes $0.016 \mathrm{~mL} \mathrm{CO}_{2} / \mathrm{min}$. At this rate, the volume of water in the device $(0.1 \mathrm{~mL})$ can be saturated with $\mathrm{CO}_{2}$ $\left(0.076 \mathrm{~mL} \mathrm{CO}_{2}\right)$ within 4.5 minutes. Up until this time, the impedance is expected to be a function of only ion due to the introduction of carbonic acid.

The second regime occurs when no additional $\mathrm{CO}_{2}$ can be dissolved in the water. At this point, any generated $\mathrm{CO}_{2}$ remains in the gas form (i.e., bubbles), which produce a higher electrical impedance due to their higher resistance and lower permittivity, compared to water. Since the size and position of bubbles over time is a stochastic process, it is reasonable to expect larger variations in electrical impedance in this regime; this trend is visible in the larger error bars of Figure 4 in the latter end of the plots. The exact transition point between the two regimes may be shorter or longer due to specific kinetics variations of the particular yeast used. In our case, the transition point occurs between 3.5 and 6.5 minutes. Therefore, by measuring the rate of change of impedance within one minute of activation, we ensure that the device readings are not affected by the stochastic variations from bubbles, but are instead directly proportional to the generation (and dissolution) of $\mathrm{CO}_{2}$. After one hour, all the DI water is evaporated or consumed, the impedance approaching its initial value in dry state.

The data from the control experiments (labeled $3^{\circ} \mathrm{C}$ in Figure 4) confirm that any possible impurities in the yeast do not significantly contribute to changes in impedance. Since the yeast cells are inactive at $3{ }^{\circ} \mathrm{C}$, any change in impedance due to only the impurities would be visible in this data set. However, aside from a slight increase in impedance within the first $30 \mathrm{~s}$ (explainable by the positive permittivity change between electrodes with water injection), the impedance does not change significantly compared to the changes observed at room temperature.

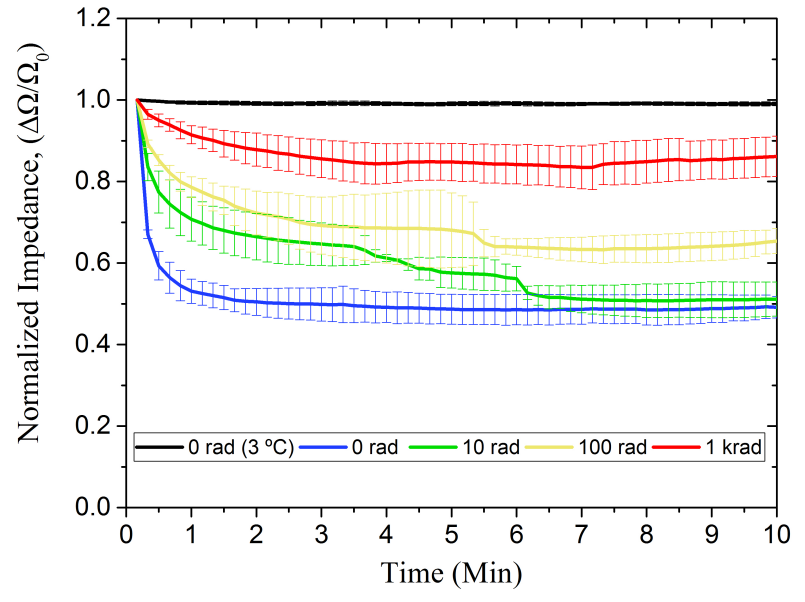

Figure 4. Change in electrical impedance of irradiated film rad sensors response to 0,10, 100, $1000 \mathrm{rad}$. The temperature controlled experiment was conducted at $3{ }^{\circ} \mathrm{C}$ while the rest were performed at room temperature.

Figure 5 shows the rate of change of impedance during the initial period, as a function of radiation dose. The data reveal a linear relationship, with the rate of change in normalized impedance after one minute of fermentation being $-0.563 \mathrm{~min}^{-1},-0.351 \mathrm{~min}^{-1}$, $-0.257 \mathrm{~min}^{-1}$, and $-0.102 \mathrm{~min}^{-1}$ for radiation doses of $0,10,100$, and $1000 \mathrm{rad}$, respectively. The average sensitivity of the sensor is $0.154 \Omega / \Omega_{0} /$ decade-rad at 1 minute after testing, Figure 5 , with an average resolution of $5 \mathrm{rad}$ during the first $10 \mathrm{rad}$.

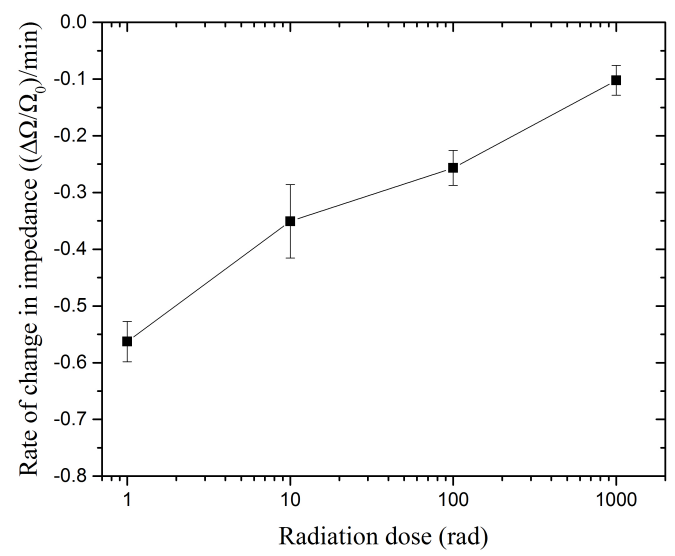

Figure 5. A linear characteristic of rate of change in impedance from irradiated sensor at $1 \mathrm{~min}$.

\section{CONCLUSIONS}

We have developed a low-cost, wearable, film-type radiation sensor that utilizes yeast as the sensing material, hence, producing an output that can be directly correlated to the DNA damage and cellular inactivation/death. Our impedance-based sensor output is a function of fermentation byproducts (i.e., generated $\mathrm{CO}_{2}$ and resulting dissolved carbonic acid) of the surviving yeast ( $\mathrm{S}$. cerevisiae) after exposure to the ionizing radiation. A prototype sensor with dimensions of $18 \times 18 \mathrm{~mm}^{2}$ shows a maximum sensitivity of $0.154 \Omega / \Omega_{0}$ decade-rad. The sensor output clearly delineates two working regimes, with the first one occurring in the 
initial few minutes, indicative of the generated ions in the water the result of the fermentation process, and the second one subsequent to the saturation of the water with the dissolved $\mathrm{CO}_{2}$, lasting much longer.

\section{ACKNOWLEDGEMENTS}

The authors thank the staff of the Birck Nanotechnology Center for their support. Travel support has been generously provided by the Transducers Research Foundation.

\section{REFERENCES}

[1] F. A. Mettler Jr., and G. L. Voelz. "Major radiation exposurewhat to expect and how to respond." New England Journal of Medicine, 346, 20, (2002), pp. 1554-1561.

[2] C.E. Land, "Estimating cancer risks from low doses of ionizing radiation", Science, 209. 4462, (1980), pp. 1197-1203.

[3] A. Martin, S. Harbison, K. Beach, and P. Cole. "An Introduction to Radiation Protection", 6E, CRC Press, (2012).

[4] M. Ehrlich, "The use of film badges for personnel monitoring", IAEA, (1962).

[5] I. Clairand, J.M. Carinou, E. Daures, J. Deboroas, J. Denoziere, M. Denoziere, L. Donadille, M. Ginjaume, C. Itie, C. Koukorava, S. Krim, A.L. Lebacq, P. Marin, L. Struelens, M. Sans-Merce, and F. Vanhavere, "Use of active personal dosimeters in interventional radiology and cardiology: Tests in laboratory conditions and recommendations-ORAMED project", Radiation Measurements, 46. 11, (2011), pp. 12521257.

[6] A. Holmes-Siedle, and L. Adams, "RADFET: a review of the use of metal-oxide-silicon devices as integrating dosimeters", International Journal of Radiation Applications and Instrumentation. Part C. Radiation Physics and Chemistry, 28. 2, (1986), pp. 235-244.
[7] E. L. Alpen, "Radiation biophysics", Academic Press, (1997).

[8] S. Tugendreich, D. E. Bassett, V. A. McKusick, M. S. Boguski, and P. Hieter, "Genes conserved in yeast and humans", Hum. Mol. Genet., vol. 3 Spec No, (1994), pp. 1509-1517.

[9] M. A. Resnick and B. S. Cox, "Yeast as an honorary mammal," Mutat. Res. - Fundam. Mol. Mech. Mutagen., vol. 451, no. 12, (2000), pp. 1-11.

[10] D. Bayrock and W. M. Ingledew, "Fluidized bed drying of baker's yeast: Moisture levels, drying rates, and viability changes during drying," Food Res. Int., vol. 30, no. 6, (1997), pp. 407-415.

[11] C. Hartmann and A. Delgado, "Numerical simulation of the mechanics of a yeast cell under high hydrostatic pressure," $J$. Biomech., vol. 37, no. (2004), pp. 977-987.

[12] T. Saeki, I. Machida, and S. Nakai, "Genetic control of diploid recovery after $\gamma$-irradiation in the yeast Saccharomyces cerevisiae," Mutation Research/Fundamental and Molecular Mechanisms of Mutagenesis, vol. 73, no. 2. (1980), pp. 251265.

[13] C. B. Bennett, L. K. Lewis, G. Karthikeyan, K. S. Lobachev, Y. H. Jin, J. F. Sterling, J. R. Snipe, and M. a Resnick, "Genes required for ionizing radiation resistance in yeast.," Nat. Genet., vol. 29, no. 4, (2001), pp. 426-434.

[14] C.K. Yoon, A. Kim, M. Ochoa, T. Parupud and B. Ziaie, "A low-cost wearble radiation sensor based on dose response viability of yeast cells", Proceedings in MEMS 2016 Shanghai, (2016).

[15] C. N. Murray, and J. P. Riley, "The solubility of gases in distilled water and sea water-IV. Carbon dioxide", In Deep Sea Research and Oceanographic Abstracts, Elsevier, Vol. 18, No. 5, (1971), pp. 533-541.

\section{CONTACT}

*C.K. Yoon, tel: +1-765-586-3020; yoon47@purdue.edu 